\title{
Introdução à Teoria Quântica de Campos: do Oscilador Harmônico ao Campo Escalar Livre
}

\author{
Rodrigo Gonçalves Pereira e Eduardo Miranda \\ Instituto de Física Gleb Wataghin, Unicamp, C. P. 6165, CEP 13083-970 - Campinas, SP
}

Recebido em 8 março, 2002. Aceito em 8 de maio, 2002.

\begin{abstract}
As teorias quânticas de campo são fundamentais em teoria de Matéria Condensada e Física de Altas Energias. Nesse artigo mostramos como um formalismo típico de teorias de campos pode ser introduzido didaticamente, generalizando a solução do oscilador harmônico para um grande número de graus de liberdade. No limite contínuo, esse método nos leva à quantização do campo de uma corda esticada vibrante e à existência dos fônons como excitações elementares ("partículas") desse campo. O procedimento pode ser usado como introdução elementar às teorias quânticas de campo através de ferramentas ensinadas em um curso de graduação de Mecânica Quântica.

Quantum field theories are central to both Condensed Matter and High-Energy Physics. In this article, we show how a typical field theoretical formalism can be pedagogically constructed by generalizing the solution of a harmonic oscillator to a large number of degrees of freedom. In the continuum limit, this method leads to the quantization of the field of a vibrating stretched string as well as to the existence of phonons as elementary excitations ("particles") of this field. The procedure can be used as an elementary introduction to quantum field theory by means of tools taught at an undergraduate course in Quantum Mechanics.
\end{abstract}

\section{Introdução}

Entendemos por campo uma função definida em todos os pontos do espaço. Por ser definido sobre um conjunto contínuo de pontos, um único campo incorpora um número infinito de graus de liberdade. O exemplo mais familiar é o campo eletromagnético, que é o centro das atenções de todo o Eletromagnetismo [1].

A proposta da Teoria Quântica de Campos é quantizar esses objetos matemáticos, assim como a Mecânica Quântica trata de quantizar as grandezas físicas relacionadas ao movimento de um número finito de partículas. A forma de fazer isso é escrever os observáveis em termos de operadores que aumentam ou diminuem o número de certas quantidades discretas no sistema, conhecidos como quanta de excitação. Tais quantidades são então identificadas com partículas elementares cujas propriedades (como massa, carga elétrica e spin) se refletem nas propriedades do campo. Por exemplo, as partículas que resultam da quantização do campo eletromagnético (mais propriamente, do quadripotencial $A^{\mu}$ ) são os fótons, que têm massa e carga nulas e spin 1. Como toda a informação sobre o número e estado das partículas pode ser resumida na descrição do estado do campo, o formalismo de teoria de campos é conveniente para tratar sistemas de muitas partículas. Mais do que isso: numa teoria rela- tivística, com possibilidade de criação e aniquilação de partículas, a função de onda de uma partícula perde o significado, e o formalismo de campos é essencial e inevitável $[2,3]$.

O objetivo deste artigo é mostrar como quantizar um campo unidimensional, correspondente a uma corda vibrante, da maneira mais pedagógica possível. Esse procedimento torna-se bastante simples quando começamos tratando um sistema discreto de osciladores acoplados. Por isso, na seção II, revisamos a solução quântica do oscilador harmônico. Na seção III, tratamos o problema de dois osciladores acoplados. Na seção IV, generalizamos a solução para $N$ osciladores acoplados, diagonalizando o Hamiltoniano através dos modos normais de vibração. Por fim, na seção V, tomamos o limite contínuo (parâmetro de rede indo a zero) e encontramos o espectro do Hamiltoniano da nossa teoria de campo.

\section{Oscilador harmônico}

O oscilador harmônico unidimensional de massa $m$ e constante de mola $C$ é regido pela Lagrangiana [4]

$$
L(x, \dot{x})=\frac{1}{2} m \dot{x}^{2}-\frac{1}{2} C x^{2},
$$


onde $x$ é a posição da partícula. O momento canonicamente conjugado a $x$ é

$$
p=\frac{\partial L}{\partial \dot{x}}=m \dot{x}
$$

O Hamiltoniano, que deve ser escrito como função de $x$ e $p$, é

$$
H(x, p)=p \dot{x}-L=\frac{p^{2}}{2 m}+\frac{1}{2} C x^{2} .
$$

A quantização do movimento da partícula é feita associando-se a $x$ e $p$ operadores Hermitianos que satisfazem a relação de comutação canônica

$$
[x, p]=i \hbar \text {. }
$$

Um estado qualquer da partícula é descrito por um ket genérico $|\psi\rangle$. Na base de autoestados de posição $\{|x\rangle\},|\psi\rangle$ é representado por uma função de onda $\psi(x)=\langle x \mid \psi\rangle$, tal que $|\psi(x)|^{2}=\psi^{*}(x) \psi(x)$ representa a densidade de probabilidade de encontrar a partícula entre $x$ e $x+d x$. Queremos encontrar as soluções da equação de Schrödinger independente do tempo

$$
H|\psi\rangle=E|\psi\rangle,
$$

onde os valores de $E$ são as energias permitidas do sistema. Para isso, definem-se os operadores adimensionais

$$
\begin{aligned}
\bar{x} & =\sqrt{\frac{m \omega}{\hbar}} x, \\
\bar{p} & =\frac{1}{\sqrt{m \hbar \omega}} p
\end{aligned}
$$

onde $\omega=\sqrt{\frac{C}{m}}$, que satisfazem

$$
[\bar{x}, \bar{p}]=i
$$

O Hamiltoniano fica então

$$
H=\frac{1}{2} \hbar \omega\left(\bar{p}^{2}+\bar{x}^{2}\right) .
$$

Definem-se os operadores $a$ e $a^{\dagger}$ na forma

$$
\begin{aligned}
a & =\frac{1}{\sqrt{2}}(\bar{x}+i \bar{p}), \\
a^{\dagger} & =\frac{1}{\sqrt{2}}(\bar{x}-i \bar{p}) .
\end{aligned}
$$

Invertendo essas relações, obtemos

$$
\begin{aligned}
\bar{x} & =\frac{1}{\sqrt{2}}\left(a+a^{\dagger}\right), \\
\bar{p} & =\frac{-i}{\sqrt{2}}\left(a-a^{\dagger}\right) .
\end{aligned}
$$

Da Eq. (8), temos

$$
\left[a, a^{\dagger}\right]=1
$$

Substituindo $\bar{x}$ e $\bar{p}$ na Eq. (9), obtemos $H$ na forma

$$
H=\hbar \omega\left(N+\frac{1}{2}\right),
$$

onde $N \equiv a^{\dagger} a$ é chamado de operador número. De (14), temos as relações de comutação entre $H$ e os operadores $a$ e $a^{\dagger}$

$$
\begin{aligned}
{[H, a] } & =-\hbar \omega a, \\
{\left[H, a^{\dagger}\right] } & =\hbar \omega a^{\dagger} .
\end{aligned}
$$

Decorre das Eq. (16) e (17) que, se $|\psi\rangle$ é um autovetor de $H$ com energia $E$, então $a^{\dagger}|\psi\rangle$ e $a|\psi\rangle$ são autovetores de $H$ com energias $E+\hbar \omega$ e $E-\hbar \omega$, respectivamente, pois

$$
\begin{aligned}
& {\left[H, a^{\dagger}\right]|\psi\rangle=\hbar \omega a^{\dagger}|\psi\rangle \Rightarrow H a^{\dagger}|\psi\rangle=(E+\hbar \omega) a^{\dagger}|\psi\rangle,} \\
& {[H, a]|\psi\rangle=-\hbar \omega a|\psi\rangle \Rightarrow H a|\psi\rangle=(E-\hbar \omega) a|\psi\rangle .}
\end{aligned}
$$

Logo, $a$ aniquila um quantum de energia $\hbar \omega$ e $a^{\dagger}$ cria o mesmo quantum. Por isso, os operadores $a$ e $a^{\dagger}$ são conhecidos como operador de aniquilação e operador de criação.

O espectro de $N$ é formado por inteiros não negativos $n=0,1,2, \ldots$ [4]. Conseqüentemente, os níveis de energia são discretos e dados por

$$
E_{n}=\hbar \omega\left(n+\frac{1}{2}\right) \quad(n=0,1,2, \ldots)
$$

A menor energia permitida é a chamada energia de ponto zero

$$
E_{0}=\frac{1}{2} \hbar \omega .
$$

O estado fundamental, denotado por $|0\rangle$, é tal que

$$
a|0\rangle=0,
$$

pois o operador a não pode criar níveis com energia menor do que $E_{0}$. O $n$-ésimo estado excitado é construído a partir do estado fundamental, aplicando-se o 
operador de criação $n$ vezes sobre o estado fundamental

$$
|n\rangle=\frac{1}{\sqrt{n !}}\left(a^{\dagger}\right)^{n}|0\rangle
$$

onde $1 / \sqrt{n !}$ é a constante de normalização, tal que $\langle n \mid n\rangle=1$. A atuação dos operadores de criação e aniquilação sobre os auto-estados do oscilador harmônico é dada por

$$
\begin{aligned}
a|n\rangle & =\sqrt{n}|n-1\rangle, \\
a^{\dagger}|n\rangle & =\sqrt{n+1}|n+1\rangle .
\end{aligned}
$$

Pode-se demonstrar que a função de onda do estado $|n\rangle$ é

$$
\psi_{n}(x)=\langle x \mid n\rangle=H_{n}(x) e^{-\frac{1}{2} \frac{m \omega}{\hbar} x^{2}},
$$

onde $H_{n}(x)$ é o polinômio de Hermite de ordem $n$.

\section{Dois osciladores acoplados}

Podemos tratar problemas que envolvem mais de uma partícula usando a equação de Schrödinger independente do tempo (5), onde $\psi$ é agora uma função das $N$ coordenadas que informam a posição de cada partícula e $E$ é a energia do sistema. Denotaremos essas posições por $u_{1}, u_{2}, \ldots, u_{N} ;$ assim, $\psi=\psi\left(u_{1}, u_{2}, \ldots, u_{N}\right) \equiv$ $\psi\left(u_{j}\right)$. Para quantizar esse sistema, precisamos conhecer o Hamiltoniano clássico em função das quantidades canonicamente conjugadas e então associar operadores a essas quantidades. Se a energia potencial depender apenas das coordenadas, a Lagrangiana será dada por $[5]$

$$
L\left[u_{j}, \dot{u}_{j}\right]=\sum_{j=1}^{N} \frac{1}{2} m_{j} \dot{u}_{j}^{2}-V\left(u_{1}, \ldots, u_{N}\right)
$$

Se $p_{j}=\partial L / \partial \dot{u}_{j}=m \dot{u}_{j}$ é o momento canonicamente conjugado a $u_{j}$, o Hamiltoniano clássico será

$$
H\left[u_{j}, p_{j}\right]=\sum_{j=1}^{N} p_{j} \dot{u}_{j}-L=\sum_{j=1}^{N} \frac{p_{j}^{2}}{2 m_{j}}+V\left(u_{1}, \ldots, u_{N}\right) .
$$

Associam-se às variáveis canônicas clássicas operadores de posição $u_{i}$ e momento $p_{i}$ que satisfazem

$$
\begin{aligned}
{\left[u_{i}, u_{j}\right] } & =\left[p_{i}, p_{j}\right]=0 \\
{\left[u_{i}, p_{j}\right] } & =i \hbar \delta_{i j}
\end{aligned}
$$

Começamos com o problema simples de duas partículas, de massas $m_{1}$ e $m_{2}$, ligadas pela energia potencial

$$
V\left(u_{1}, u_{2}\right)=\frac{1}{2} C\left(u_{2}-u_{1}\right)^{2},
$$

que corresponde ao problema clássico de duas massas acopladas por uma mola de constante $C$. O Hamiltoniano do sistema é

$$
H=\frac{p_{1}^{2}}{2 m_{1}}+\frac{p_{2}^{2}}{2 m_{2}}+\frac{1}{2} C\left(u_{2}-u_{1}\right)^{2} .
$$

É conveniente reescrever a Eq. (32) em termos das novas coordenadas

$$
\begin{aligned}
v & =u_{2}-u_{1} \\
w & =\frac{m_{1} u_{1}+m_{2} u_{2}}{M},
\end{aligned}
$$

onde $M \equiv m_{1}+m_{2}$. As coordenadas $v$ e $w$ são facilmente reconhecidas como a coordenada relativa entre $m_{1}$ e $m_{2}$ e a coordenada do centro de massa do sistema, respectivamente. Os momentos conjugados a $v \mathrm{e}$ $w$ são

$$
\begin{aligned}
& p_{v}=\frac{m_{1} p_{2}-m_{2} p_{1}}{M}, \\
& p_{w}=p_{1}+p_{2} .
\end{aligned}
$$

Com essa transformação, obtém-se

$$
H=\frac{p_{v}^{2}}{2 \mu}+\frac{1}{2} C v^{2}+\frac{p_{w}^{2}}{2 M}
$$

onde $\mu \equiv m_{1} m_{2} /\left(m_{1}+m_{2}\right)$ é a massa reduzida. O Hamiltoniano (37) é separável nas variáveis $v$ e $w$; isso significa que a função de onda e a energia dos estados estacionários podem ser escritas na forma

$$
\begin{aligned}
\psi(v, w) & =\varphi(v) \chi(w) \\
E & =E_{v}+E_{w} .
\end{aligned}
$$

A equação em $w$ é

$$
\frac{p_{w}^{2}}{2 M} \chi(w)=E_{w} \chi(w)
$$

e corresponde ao movimento de uma partícula livre de massa $M$ com energia cinética $E_{w}$. Definindo-se $k$ tal que

$$
E_{w}=\frac{\hbar^{2} k^{2}}{2 M},
$$

a solução é uma onda plana do tipo

$$
\chi(w)=e^{i k w} .
$$

Já a equação em $v$ é

$$
\left(\frac{p_{v}^{2}}{2 \mu}+\frac{1}{2} C v^{2}\right) \varphi(v)=E_{v} \varphi(v) .
$$

Comparando-se com (3), vê-se que essa é a equação de Schrödinger de um oscilador harmônico com massa $\mu$ e freqüência $\omega=\sqrt{C / \mu}$. Da seção 6 , sabemos que os 
níveis de energia são quantizados como em $(20)$ e $\varphi_{n}(v)$ é dada por (26). Logo, a energia total é

$$
E=\frac{\hbar^{2} k^{2}}{2 M}+\hbar \omega\left(n+\frac{1}{2}\right) .
$$

O método empregado acima sugere uma generalização que permite tratar o caso de $N$ corpos. O fato fundamental é que a equação de Schrödinger independente do tempo torna-se separável quando se escolhe um sistema de coordenadas conveniente. No caso considerado, a coordenada $v$ corresponde a uma forma de movimento em que tanto $m_{1}$ quanto $m_{2}$ vibravam com a freqüência $\omega$; por sua vez, $w$ corresponde à translação conjunta de $m_{1}$ e $m_{2}$ - o que pode ser encarado como uma vibração com freqüência $\omega=0$. Cada uma dessas formas de movimento, em que todas as partículas vibram com a mesma freqüência, é chamada de um modo normal do sistema.

Temos, então, uma estratégia para resolver o problema quântico de $N$ osciladores acoplados: encontrar os modos normais do sistema pela mesma técnica empregada no caso clássico, separar a equação de Schrödinger nas coordenadas dos modos normais e reduzir o problema a $N$ equações de osciladores harmônicos simples, cuja solução já conhecemos. Essa estratégia será seguida na próxima Seção.

\section{N osciladores acoplados}

\section{IV.1 Diagonalização do Hamiltoniano}

Vamos considerar agora o problema de $N$ massas acopladas por $N$ molas (Fig. 1). Por simplicidade, assumimos que todas as massas são iguais $\left(m_{i}=m\right)$ e todas as molas têm a mesma constante $C$. Assim, a cada mola está associada uma energia potencial da forma

$$
V_{j}=\frac{1}{2} C\left(u_{j+1}-u_{j}\right)^{2} .
$$

Além disso, impomos condições periódicas de contorno

$$
u_{j+N}(t)=u_{j}(t)
$$

A relação (46) pode ser visualizada como a construção de uma cadeia de $N$ partículas acopladas por molas em que se liga a última delas à primeira por outra mola, formando um círculo fechado com $N$ molas. As condições de periódicas de contorno, embora não essenciais ao desenvolvimento que se segue, facilitam sobremaneira os cálculos.

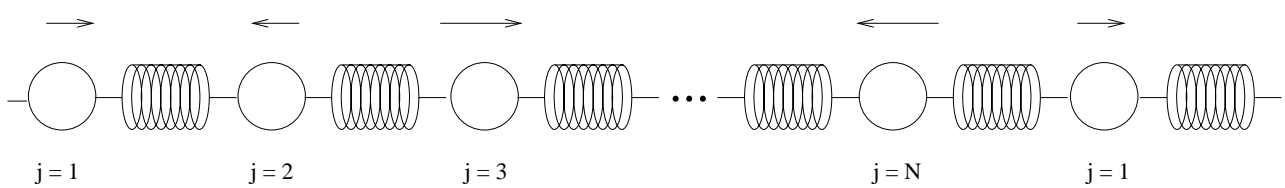

Figura 1. N osciladores harmônicos acoplados com condições periódicas de contorno.

A Lagrangiana do sistema é

$$
\begin{aligned}
L\left[u_{j}, \dot{u}_{j}\right] & =\sum_{j=1}^{N}\left[\frac{1}{2} m \dot{u}_{j}^{2}-\frac{1}{2} C\left(u_{j+1}-u_{j}\right)^{2}\right] \\
& =\frac{1}{2} m \sum_{j=1}^{N}\left[\dot{u}_{j}^{2}-\frac{C}{m}\left(u_{j+1}^{2}-2 u_{j} u_{j+1}+u_{j}^{2}\right)\right] .
\end{aligned}
$$

O Hamiltoniano é

$$
H\left[u_{j}, p_{j}\right]=\sum_{j=1}^{N}\left[\frac{p_{j}^{2}}{2 m}-\frac{1}{2} C\left(u_{j+1}-u_{j}\right)^{2}\right],
$$

onde

$$
p_{j}=\frac{\partial L}{\partial \dot{q}_{j}}=m \dot{u}_{j}
$$

O termo da energia potencial pode ser simplificado notando que cada $u_{j}^{2}$ aparece duas vezes na soma. Temos, então,

$$
L\left[u_{j}, \dot{u}_{j}\right]=\frac{1}{2} m \sum_{j=1}^{N}\left[\dot{u}_{j}^{2}-\frac{C}{m}\left(2 u_{j}^{2}-2 u_{j} u_{j+1}\right)\right]
$$


Definindo o vetor de coordenadas

$$
u=\left(\begin{array}{l}
u_{1} \\
u_{2} \\
\vdots \\
u_{N}
\end{array}\right)
$$

podemos escrever $L\left[u_{j}, \dot{u}_{j}\right]$ na forma de um produto de matrizes

$$
L=\frac{1}{2} m\left[\dot{u}^{t} \dot{u}-\frac{C}{m} u^{t} A u\right]
$$

onde $A$ é a matriz $N \times N$ dos coeficientes $a_{i j}$ de $u_{i} u_{j}$

$$
A=\left(\begin{array}{rrrrr}
2 & -1 & 0 & \cdots & -1 \\
-1 & 2 & -1 & & \\
0 & -1 & 2 & & \vdots \\
\vdots & & & \ddots & \\
-1 & & \ldots & & 2
\end{array}\right)
$$

e o superíndice $t$ denota a transposição da matriz.

Como $A$ é simétrica, existe uma mudança de coordenadas dada por uma matriz ortogonal $G$ que diagonaliza $A$, isto é

$$
u=G q
$$

$\operatorname{com} G$ tal que

$$
\begin{aligned}
G^{t} & =G^{-1}, \\
G^{t} A G & =D, \\
d_{i j} & =\alpha_{i}^{2} \delta_{i j},
\end{aligned}
$$

onde $d_{i j}$ são os elementos da matriz $D$. Os autovalores $\alpha_{i}^{2}$ de $A$ são todos não negativos porque $u=0$ é uma configuração de equilíbrio estável do sistema. Substituindo (55) na Eq. (53) e usando (57), obtemos

$$
L\left[q_{j}, \dot{q}_{j}\right]=\frac{1}{2} m\left[\dot{q}^{t} \dot{q}-\frac{C}{m} q^{t} D q\right]=\sum_{j=1}^{N}\left[\frac{1}{2} m \dot{q}_{j}^{2}-\frac{1}{2} C \alpha_{j}^{2} q_{j}^{2}\right] .
$$

O Hamiltoniano associado é

$$
H\left[q_{j}, \Pi_{j}\right]=\sum_{j=1}^{N}\left[\frac{\Pi_{j}^{2}}{2 m}+\frac{1}{2} C \alpha_{j}^{2} q_{j}^{2}\right],
$$

onde $\Pi_{j}=m \dot{q}_{j}$. A transformação ortogonal assegura que $q_{j}$ e $\Pi_{j}$ são também canonicamente conjugados, isto é,

$$
\left[q_{i}, \Pi_{j}\right]=i \hbar \delta_{i j}
$$

Notamos que o Hamiltoniano (60) é separável nas variáveis $q_{j}$, que descrevem os modos normais de vibração do sistema. Cada modo possui uma freqüência associada

$$
\omega_{j}=\alpha_{j} \sqrt{\frac{C}{m}} .
$$

Logo, a equação de Schrödinger admite soluções da forma

$$
\psi=Q_{1}^{n_{1}}\left(q_{1}\right) Q_{2}^{n_{2}}\left(q_{2}\right) \ldots Q_{N}^{n_{N}}\left(q_{n}\right),
$$

onde cada $Q_{j}^{n_{j}}$ é dada por (26), com autovalor

$$
E_{j}=\hbar \omega_{j}\left(n_{j}+\frac{1}{2}\right)
$$

Portanto, a energia total do sistema é quantizada em termos das freqüências dos modos normais

$$
E=\sum_{j=1}^{N} E_{j}=\sum_{j=1}^{N} \hbar \omega_{j}\left(n_{j}+\frac{1}{2}\right)
$$

A energia de ponto zero é

$$
E_{0}=\sum_{j=1}^{N} \frac{1}{2} \hbar \omega_{j}
$$

\section{IV.2 A relação de dispersão}

As freqüências $\omega_{j}$, das quais dependem os níveis de energia do sistema com $N$ osciladores, são as freqüências clássicas dos modos normais de vibração. Para encontrá-las, consideramos as equações de movimento derivadas das equações de Euler-Lagrange [5]

$$
\frac{d}{d t}\left(\frac{\partial L}{\partial \dot{u}_{j}}\right)-\frac{\partial L}{\partial u_{j}}=0
$$

que nos levam a

$$
m \ddot{u}_{j}=C\left(u_{j+1}-u_{j}\right)-C\left(u_{j}-u_{j-1}\right) .
$$

$\mathrm{Na}$ Eq. (68), cada $u_{j}$ se acopla com os dois primeiros vizinhos. Quando o sistema está num modo normal, todas as partículas oscilam com a mesma freqüência. 
Além disso, a invariância da Lagrangiana por uma translação de $j \rightarrow j+1$ assegura que exista uma solução do tipo onda plana (usando notação complexa por conveniência)

$$
u_{j}(t)=a_{j} e^{i(k j h-\omega t)},
$$

onde $a_{j}$ é uma amplitude complexa de vibração, $k=$ $2 \pi / \lambda$ é o número de onda e $h$ é a separação de equilíbrio entre dois sítios vizinhos (parâmetro de rede). Logo, a posição de equilíbrio da massa de índice $j$ é $x_{j}=j h$. Substituindo (69) em (68), encontramos as freqüências normais de oscilação, também conhecida como a relação de dispersão do sistema de $\mathrm{N}$ osciladores acoplados

$$
\omega(k)=\sqrt{\frac{4 C}{m}}\left|\sin \left(\frac{k h}{2}\right)\right| .
$$

De Eq. (70), podemos ver que qualquer intervalo de $k h$ de amplitude $2 \pi$ é capaz de fornecer todos os valores de $\omega$ possíveis. É costume escolher o intervalo simétrico

$$
-\frac{\pi}{h} \leq k \leq \frac{\pi}{h}
$$

conhecido como primeira zona de Brillouin [6]. Observe que a existência de um $k$ máximo está ligada ao fato da separação entre as massas ser finita $(h>0)$. Além disso, a Eq. (70) implica que há uma freqüência máxima que ocorre justamente para $|k|=\pi / h$ e é dada por

$$
\omega_{M}=\sqrt{\frac{4 C}{m}} .
$$

A relação de dispersão (70) é mostrada na Fig. 2.

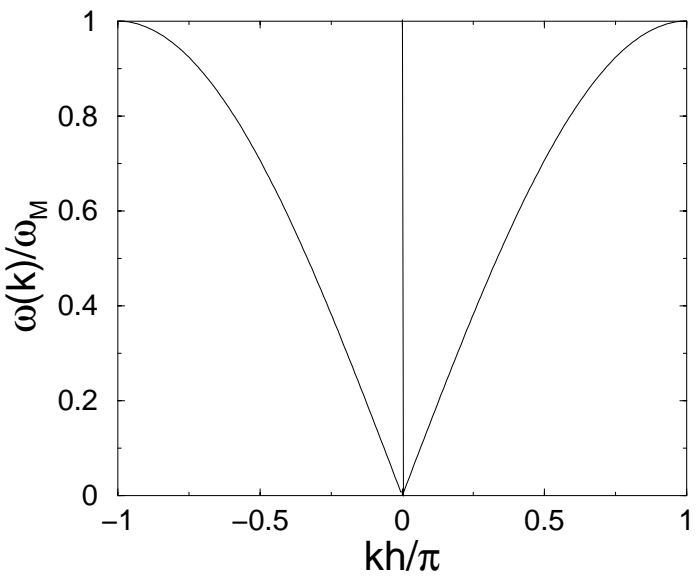

Figura 2. Relação de dispersão de $\mathrm{N}$ osciladores harmônicos acoplados.

A velocidade de fase da onda com vetor de onda $k$ é dada por

$$
v(k)=\frac{\omega(k)}{k}=\frac{\omega_{M}}{k}\left|\sin \left(\frac{k h}{2}\right)\right| .
$$

Para $k h \ll 1$ (comprimentos de onda muito maiores que o parâmetro de rede), a velocidade de fase é aproximadamente constante

$$
\begin{aligned}
\omega & \simeq \omega_{M} \frac{|k| h}{2} \\
\Rightarrow v & \simeq \pm h \sqrt{\frac{C}{m}}
\end{aligned}
$$

As freqüências dos $N$ modos normais surgem quando exigimos que a solução (69) satisfaça as condições periódicas de contorno, o que ocorre se

$$
e^{i k j h}=e^{i k h(j+N)} \text {. }
$$

Isso reduz os valores de $k$ àqueles, tal que

$$
k_{j}=\frac{2 j \pi}{N h}=j \frac{2 \pi}{L},
$$

onde $j=0, \pm 1, \pm 2, \ldots$ e $L=N h$ é o comprimento total da rede. Os $k_{j}$ são separados pela quantidade $\Delta k=k_{j+1}-k_{j}=2 \pi / L$. Se quisermos limitar os valores de $k$ à primeira zona de Brillouin (71), devemos tomar

$$
j= \begin{cases}-\frac{N}{2}+1, \cdots, 0, \cdots, \frac{N}{2} & N \text { par } \\ \frac{-N+1}{2}, \cdots, 0, \cdots, \frac{N-1}{2} & N \text { impar. }\end{cases}
$$

As freqüências $\omega_{j}$ são finalmente obtidas substituindo-se $k_{j}$ na relação de dispersão

$$
\omega_{j}=\omega_{M}\left|\sin \left(\frac{j \pi}{N}\right)\right| .
$$

Vê-se que as freqüências dos modos normais são degeneradas, pois $\omega_{j}=\omega_{-j}$. De fato, as soluções com $k_{j}$ e $k_{-j}=-k_{j}$ correspondem a ondas de mesma freqüência que se propagam pela rede em sentidos opostos. $\mathrm{O}$ modo de freqüência $\omega=0$ é o modo de translação livre do sistema, como discutimos na seção III.

\section{O limite contínuo}

\section{V.1 Lagrangiana e Hamiltoniano da corda}

Um sistema contínuo pode ser encarado como o limite de um sistema de partículas quando o número de graus de liberdade tende a infinito. Toma-se, então, o cuidado de fazer a correspondência correta entre as grandezas características do sistema discreto e suas análogas no contínuo. No caso que temos tratado, o problema de $N$ partículas ligadas por molas reduz-se, quando $N \rightarrow \infty$ e $h \rightarrow 0$ (mantendo $L=N h$ fixo), ao problema de uma corda contínua que vibra longitudinalmente. A dinâmica da corda depende da densidade linear de massa e de uma constante relacionada 
à sua elasticidade. $\mathrm{O}$ índice $j$ em $u_{j}(t)$ deve tornar-se contínuo e relacionado à coordenada $x$ do ponto sobre a corda

$$
u_{j}(t) \rightarrow u(x=j h, t) .
$$

A grandeza $u(x, t)$ define, então, uma função contínua no domínio $0 \leq x \leq L$, ou seja, $u(x, t)$ é um campo escalar unidimensional, que descreve o deslocamento de cada ponto da corda em relação à sua posição de equiíbrio.

A propriedade de inércia da corda é dada pela densidade linear de massa

$$
\sigma=\lim _{h \rightarrow 0} \frac{m}{h}=\frac{M}{L},
$$

onde $M$ é a massa total da corda. A energia cinética do sistema discreto em (48) pode ser reescrita na forma

$$
T\left[\dot{u}_{j}\right]=\sum_{j} h \frac{1}{2} \frac{m}{h} \dot{u}_{j}^{2}
$$

No limite contínuo, substituímos $\sum_{j} h$ por $\int d x$ e $m / h$ por $\sigma$, obtendo

$$
T[\dot{u}(x)]=\int d x\left[\frac{1}{2} \sigma \dot{u}^{2}(x)\right] .
$$

onde a integral é calculada no intervalo $-L / 2 \leq x \leq$ $L / 2$.

Já a energia potencial em (48) fica

$$
V\left[\dot{u}_{j}\right]=\sum_{j} h \frac{1}{2} C h\left(\frac{u_{j+1}-u_{j}}{h}\right)^{2} .
$$

Identificando

$$
\begin{aligned}
C h & \rightarrow \tau, \\
\frac{u_{j+1}-u_{j}}{h} & \rightarrow \lim _{h \rightarrow 0} \frac{u(x+h)-u(x)}{h}=\partial_{x} u(x)(86)
\end{aligned}
$$

encontramos que a energia potencial da corda é

$$
V\left[\partial_{x} u(x)\right]=\int d x\left[\frac{1}{2} \tau\left(\partial_{x} u(x)\right)^{2}\right] .
$$

A Lagrangiana do sistema contínuo é, então,

$$
\begin{aligned}
L\left[u(x), \dot{u}(x), \partial_{x} u(x)\right] & =\int d x\left[\frac{1}{2} \sigma \dot{u}^{2}(x)-\frac{1}{2} \tau\left(\partial_{x} u(x)\right)^{2}\right] \\
& =\int d x \mathcal{L}(x)
\end{aligned}
$$

onde

$$
\mathcal{L}(x)=\frac{1}{2} \sigma \dot{u}^{2}(x)-\frac{1}{2} \tau\left(\partial_{x} u(x)\right)^{2}
$$

é chamada de densidade de Lagrangiana. Note que a Lagrangiana, que era uma função de várias variáveis no caso discreto, torna-se agora um funcional de $u(x)$, $\dot{u}(x)$ e $\partial_{x} u(x)$, ou seja, um novo tipo de função que, nesse caso, leva funções reais pertencentes ao conjunto $\mathbb{F}$ ao conjunto dos reais

$$
L\left[u(x), \dot{u}(x), \partial_{x} u(x)\right]: \mathbb{F} \rightarrow \mathbb{R} .
$$

O momento conjugado a $u(x)$ é dado pela derivada parcial [5]

$$
\Pi(x)=\frac{\partial \mathcal{L}(x)}{\partial \dot{u}(x)}=\sigma \dot{u}(x),
$$

que é o análogo contínuo de (50). O Hamiltoniano é, então,

$$
H[u, \Pi]=\int d x\left[\frac{\Pi^{2}(x)}{2 \sigma}+\frac{1}{2} \tau\left(\partial_{x} u(x)\right)^{2}\right] .
$$

O análogo da equação de movimento (68) para a corda contínua é a equação de onda, que decorre da equação de Euler-Lagrange para variáveis contínuas [5]

$$
\frac{\partial}{\partial t}\left(\frac{\partial \mathcal{L}}{\partial_{t} u(x)}\right)+\frac{\partial}{\partial x}\left(\frac{\partial \mathcal{L}}{\partial_{x} u(x)}\right)-\frac{\partial \mathcal{L}}{\partial u(x)}=0 .
$$

Note a presença de um novo termo, devido à dependência de $\mathcal{L} \operatorname{com} \partial_{x} u$. Obtemos, então,

$$
\partial_{x}^{2} u(x, t)-\frac{1}{c^{2}} \partial_{t}^{2} u(x, t)=0
$$

onde $c=\sqrt{\tau / \sigma}$ é a velocidade da onda, que é constante. Esse é exatamente o limite $h \rightarrow 0$ na relação de dispersão (70), que se reduz a

$$
\omega_{j}=\omega\left(k_{j}\right)=c\left|k_{j}\right|
$$

\section{V.2 Diagonalização do Hamiltoniano da corda}

No limite contínuo, a função de onda de $N$ coordenadas $\psi\left(u_{j}\right)$ é substituída por um funcional de onda $\psi=\psi[u(x)]$, que descreve o estado do sistema é 
solução da equação de Schrödinger com o Hamiltoniano dado por (93). Para quantizar o Hamiltoniano em analogia com o caso discreto da Eq. (30), impõe-se que os operadores $u(x)$ e $\Pi(x)$ satisfazem as relações de comutação canônicas

$$
\begin{aligned}
{\left[u(x), u\left(x^{\prime}\right)\right] } & =\left[\Pi(x), \Pi\left(x^{\prime}\right)\right]=0, \\
{\left[u(x), \Pi\left(x^{\prime}\right)\right] } & =i \hbar \delta\left(x-x^{\prime}\right),
\end{aligned}
$$

onde $\delta(x)$ é a função delta de Dirac, que pode ser encarada com o limite contínuo do delta de Kronecker em (30).

Como no caso discreto, a estratégia é escrever $H$ em termos das coordenadas dos modos normais para obter um conjunto de osciladores harmônicos de ener- gia quantizada. Introduzimos $q_{j}$ como a amplitude do modo de número de onda $k_{j}$

$$
u(x)=\sum_{j} e^{i k_{j} x} q_{j}
$$

onde os $k_{j}$ permitidos são $k_{j}=j 2 \pi / L$. Multiplicando os dois lados da Eq. (99) por $e^{-i k_{l} x}$ e integrando sobre o comprimento da corda, obtemos a relação inversa

$$
q_{j}=\frac{1}{L} \int d x e^{-i k_{j} x} u(x)
$$

Como $u(x)$ é real, segue da Eq. (100) que $q_{n}$ é, em geral, complexo e $q_{n}^{*}=q_{-n}$. De (99), temos que

$$
\begin{aligned}
\int d x[\dot{u}(x)]^{2} & =\sum_{j} \sum_{l} \dot{q}_{j} \dot{q}_{l} \int d x e^{i\left(k_{j}+k_{l}\right) x} \\
& =L \sum_{j} \sum_{l} \dot{q}_{j} \dot{q}_{l} \delta_{j,-l}=L \sum_{j} \dot{q}_{j} \dot{q}_{-j} .
\end{aligned}
$$

Do mesmo modo,

$$
\int d x\left(\partial_{x} u(x)\right)^{2}=L \sum_{j} k_{j}^{2} q_{j} q_{-j}
$$

e a Lagrangiana pode ser escrita

$$
L=\sum_{j}\left\{\frac{1}{2} M \dot{q}_{j} \dot{q}_{-j}-\frac{1}{2} M \omega_{j}^{2} \dot{q}_{j} \dot{q}_{-j}\right\},
$$

onde substituímos $M=\sigma L$ e $\tau k_{j}^{2}=\sigma \omega_{j}^{2}$.

$\mathrm{O}$ momento conjugado a $q_{j}$ é

$$
\Pi_{j}=\frac{\partial L}{\partial \dot{q}_{j}}=M \dot{q}_{-j},
$$

lembrando que cada $q_{j}$ aparece duas vezes no somatório em (104). É fácil ver que $\Pi_{j}$ se relaciona com $\Pi(x)$ definido na Eq. (92) através das relações

$$
\begin{aligned}
\Pi(x) & =\frac{1}{L} \sum_{j} e^{i k_{j} x} \Pi_{-j}, \\
\Pi_{j} & =\int d x e^{i k_{j} x} \Pi(x) .
\end{aligned}
$$

Como no caso de $q_{j}$, temos que $\Pi_{j}^{*}=\Pi_{-j}$ porque $\Pi(x)$ é real. A relação de comutação entre os operadores $q_{j}$ e $\Pi_{j}$ pode ser obtida a partir da relação entre $u(x)$ e $\Pi\left(x^{\prime}\right)$ e das transformações (100) e (107)

$$
\left[q_{i}, \Pi_{j}\right]=\frac{1}{L} \int d x \int d x^{\prime}\left[u(x), \Pi\left(x^{\prime}\right)\right] e^{i\left(k_{j}-k_{i}\right) x}=i \hbar \delta_{i j},
$$

que tem também uma forma canônica (porém discreta).

O Hamiltoniano é dado por

$$
H=\sum_{j}\left\{\frac{\Pi_{j} \Pi_{-j}}{2 M}+\frac{1}{2} M \omega_{j}^{2} q_{j} q_{-j}\right\} .
$$

Vamos considerar o limite da corda infinita $L \rightarrow \infty$ (às vezes também chamado de limite termodinâmico). Uma vez que a separação entre os $k_{j}$ permitidos é $\Delta k=2 \pi / L$, o limite $L \rightarrow \infty$ faz $\Delta k \rightarrow 0$, isto é, o conjunto de $k$ 's permitidos torna-se contínuo, assim como a variável $x$. Por isso, denotamos cada modo por $q(k), k \in \mathbb{R}$. Escrevendo a Eq. (99) na forma

$$
u(x)=\frac{1}{\sqrt{2 \pi}} \sum_{j} \Delta k e^{i k_{j} x} \frac{q_{j}}{\sqrt{2 \pi} / L},
$$

e supondo que, no limite $\Delta k \rightarrow 0$, a seguinte expressão convirja

$$
\frac{q_{j} L}{\sqrt{2 \pi}} \rightarrow q(k)
$$

obtemos a relação

$$
u(x)=\frac{1}{\sqrt{2 \pi}} \int_{-\infty}^{+\infty} d k e^{i k x} q(k) .
$$

Logo, $q(k)$ é a transformada de Fourier de $u(x)$. Analogamente, o momento $\Pi(k)$ é tal que

$$
\Pi(x)=\frac{1}{\sqrt{2 \pi}} \int d k e^{i k x} \Pi(-k),
$$


e a relação de comutação para os operadores com índice contínuo é

$$
\left[q(k), \Pi\left(k^{\prime}\right)\right]=i \hbar \delta\left(k-k^{\prime}\right)
$$

A Lagrangiana e o Hamiltoniano em função de $q(k)$ ficam

$$
\begin{aligned}
L & =\int d k\left\{\frac{1}{2} \sigma \dot{q}(k) \dot{q}(-k)-\frac{1}{2} \sigma \omega^{2}(k) q(k) q(-k)\right\} \\
H & =\int d k\left\{\frac{\Pi(k) \Pi(-k)}{2 \sigma}+\frac{1}{2} \sigma \omega^{2}(k) q(k) q(-k)\right\}
\end{aligned}
$$

$\operatorname{com} \omega(k)=c|k|$.

\section{V.3 Fônons}

Basta comparar com a Eq. (60) para perceber que o Hamiltoniano da Eq. (116) é um soma sobre todos os modos $k$ de operadores não-Hermitianos $q(k)$ e $\Pi(k)$. Logo, o espectro de $H$ deve ser dado pelo número de excitações em cada modo de freqüência $\omega(k)$. Para mostrar isso, introduzem-se os operadores

$$
\begin{aligned}
\hat{q}(k) & =\sqrt{\frac{\sigma \omega(k)}{\hbar}} q(k), \\
\hat{\Pi}(k) & =\frac{1}{\sqrt{\sigma \hbar \omega(k)}} \Pi(k),
\end{aligned}
$$

que satisfazem a relação de comutação

$$
\left[\hat{q}(k), \hat{\Pi}\left(k^{\prime}\right)\right]=i \delta\left(k-k^{\prime}\right)
$$

Em analogia com (10) e (11), definimos

$$
\begin{aligned}
a(k) & =\frac{1}{\sqrt{2}}(\hat{q}(k)+i \hat{\Pi}(-k)), \\
a^{\dagger}(k) & =\frac{1}{\sqrt{2}}(\hat{q}(-k)-i \hat{\Pi}(k)),
\end{aligned}
$$

que, por sua vez, satisfazem

$$
\left[a(k), a^{\dagger}\left(k^{\prime}\right)\right]=\delta\left(k-k^{\prime}\right) .
$$

O Hamiltoniano da corda pode ser colocado na forma

$$
H=\int d k \hbar \omega(k)\left\{a^{\dagger}(k) a(k)+\frac{1}{2} \delta(0)\right\} .
$$

Verifica-se então que

$$
\begin{aligned}
{[H, a(k)] } & =-\hbar \omega(k) a(k), \\
{\left[H, a^{\dagger}(k)\right] } & =\hbar \omega(k) a^{\dagger}(k) .
\end{aligned}
$$

A interpretação é a esperada: os estados excitados são produzidos pela ação de operadores de criação e destruição de um número inteiro de quanta de energia $\hbar \omega(k)$. A diferença é que, neste caso, o espectro de frequiências $\omega(k)$ é contínuo. O estado fundamental $|0\rangle$ é obtido da condição

$$
a(k)|0\rangle=0,
$$

que deve ser válida para todo $k$. De acordo com a Eq. (123), a energia do estado fundamental da corda infinita é

$$
E_{0}=\int d k \frac{1}{2} \hbar \omega(k) \delta(0) .
$$

A divergência na energia do estado fundamental é comum numa teoria de campos (que lida com infinitos graus de liberdade) e aparece aqui por dois motivos. Em primeiro lugar, a integral é feita sobre todos os modos com $k$ de $-\infty$ a $+\infty$ e, como cada modo possui energia de ponto zero finita, o resultado deve ser infinito. Essa divergência pode ser corrigida introduzindo-se uma distância mínima no sistema (como um parâmetro de rede), que estabelece um $k$ máximo; tal procedimento é conhecido como regularização do ultravioleta, porque lida com pequenos comprimentos de onda. Em segundo lugar, os modos de vibração da corda infinita são contínuos; daí a origem do fator $\delta(0)$ em $E_{0}$. A solução para esse problema é justamente introduzir um tamanho finito $L$ para a corda, o que discretiza e impõe um valor mínimo para os $k$ 's. Essa é uma regularização do infravermelho (grandes comprimentos de onda). A interpretação física dessas divergências é clara: elas representam a energia necessária para a criação do número infinito de graus de liberdade do sistema (energia de criação da corda). Mesmo sem qualquer regularização, o que realmente interessa são as diferenças de energia entre os níveis, não a energia total de criação do sistema. Essas energias de excitação são dadas pelos quanta $\hbar \omega(k)$ e são finitas. O Hamiltoniano associado à energia medida a partir do estado fundamental pode, então, ser escrito

$$
H=\int d k \hbar c|k| a^{\dagger}(k) a(k) .
$$


Os estados excitados com $n_{r}$ excitações de momento $k_{r}, n_{s}$ excitações de momento $k_{s}$, etc, são construídos pela ação dos operadores $a^{\dagger}$

$$
\left|n_{r} n_{s} \ldots n_{u}\right\rangle=\frac{\left(a_{r}^{\dagger}\right)^{n_{r}}\left(a_{s}^{\dagger}\right)^{n_{s}} \ldots\left(a_{u}^{\dagger}\right)^{n_{u}}}{\sqrt{n_{r} ! n_{s} ! \ldots n_{u} !}}|0\rangle .
$$

Esse resultado mostra que é possível descrever o estado do sistema, que é vinculado ao campo escalar $u(x)$, através da criação e destruição de quanta de vibração da corda. As transições entre estados excitados acontecem quando há absorção ou emissão dessas quantidades discretas de energia por parte do sistema. Podemos levar essa interpretação um pouco mais longe e identificar esses quanta com partículas, usualmente chamadas fônons, que são caracterizadas pela energia $E(k)=\hbar \omega(k)$ e pelo momento $p(k)=\hbar k$ (segundo as relações de Einstein-De Broglie). Para que um estado excitado fique bem caracterizado, é suficiente informar o número de fônons de cada modo normal criados sobre o estado fundamental. Esses números são justamente o que se obtém aplicando-se os operadores número $N(k)$ $\equiv a^{\dagger}(k) a(k)$ sobre os autovetores de $H$. Desse ponto de vista, o estado fundamental $|0\rangle$ é um estado que não contém nenhum fônon e, por isso, é freqüentemente chamado de vácuo. Além de energia e momento, podese levar adiante a analogia com uma partícula material e se perguntar se um fônon tem uma massa de repouso associada. Dado que $E=\hbar c|k|=c|p|$, se considerarmos a expressão relativística para a energia total

$$
E=\sqrt{\left(m_{0} c^{2}\right)^{2}+(p c)^{2}}
$$

concluiremos que $m_{0}=0$, ou seja, um fônon tem massa de repouso nula.

\section{Conclusões}

O problema de quantização da corda contínua mostra que é viável introduzir uma teoria quântica de campo usando-se um sistema mecânico familiar e intuitivo para alunos de graduação. A linha seguida foi a de generalizar, passo a passo, a solução do oscilador harmônico de 1 para $N$ e de $N$ para infinitos graus de liberdade. Vimos que, nas coordenadas dos modos normais, a corda equivale a um conjunto de osciladores desacoplados de energia quantizada. Os quanta de vibração, aos quais se atribui identidade de partículas, são os fônons estudados nos cursos de Estado Sólido. A teoria de campo desenvolvida é uma teoria de campo escalar livre, pois os modos (ou fônons) são não interagentes. As interações são introduzidas através de termos não quadráticos nos campos na expressão da Lagrangiana ou da Hamiltoniana.

Além disso, esse problema ilustra um procedimento comum a outras teorias de campo que prescindem de analogia mecânica. Em tais casos, constrói-se uma Lagrangiana que fornece a equação apropriada - o que significa incorporar as propriedades das partículas nas simetrias do campo - para então quantizar o sistema impondo relações de comutação entre os operadores.

Os autores agradecem à FAPESP pelo apoio financeiro $(00 / 05413-1)$.

\section{Referências}

[1] L. Landau e E. Lifshitz, The Classical Theory of Fields, Addison-Wesley, Reading, Mass (1951).

[2] M. E. Peskin e D. V. Schroeder, An Introduction to Quantum Field Theory, Addison Wesley, Reading, Mass (1995)

[3] N. N. Bogoliubov, Quantum Fields, Benjamin/ Cumming, Reading, Mass. (1983).

[4] Cohen-Tannoudji, Quantum Mechanics, Volume I, John Wiley \& Sons, New York (1977). Chapter V.

[5] H. Goldstein, Classical Mechanics, Second edition, Addison-Wesley, Reading, Mass (1980).

[6] C. Kittel, Introduction to Solid State Physics, Seventh edition, John Wiley \& Sons, New York (1996). Chapter 4 . 